\title{
BOUNDARY INTEGRAL EQUATIONS OF DYNAMICS PROBLEMS FOR MULTI-CONNECTED THERMOELASTIC SEMI-PLANE WITH A FREE BOUNDARY
}

\author{
LYUDMILA A. ALEXEYEVA ${ }^{1} \&$ BAKHYT N. ALIPOVA ${ }^{2,3 *}$ \\ ${ }^{1}$ Institute of Mathematics and Mathematical Modeling of MES RK, Kazakhstan. \\ ${ }^{2}$ International University of Information Technology, Kazakhstan, Samara University, Russia. \\ ${ }^{3}$ Al-Farabi Kazakh National University, Kazakhstan.
}

\begin{abstract}
The dynamics of multi-connected thermoelastic semiplane with the non-stationary power source and thermal effects by using of a model of coupled thermoelasticity is investigated. Green's tensor in the space of the Laplace transforms in time describes the displacements of medium under the effect of the impulse concentrated power and thermal sources. The generalized solution of the problem of the dynamics of thermoelastic semiplane with the free boundary under the effect of arbitrary mass forces and thermal sources in 2D-case is built.
\end{abstract}

Keywords: dynamics, green's tensor, semiplane, the laplace transformation, thermoelasticity

\section{INTRODUCTION}

The various mathematical models of Mechanics of Deformable Solid are used to calculate the real properties of rock mass by the study of seismic processes in the Earth's crust. The most studied processes are wave propagation and diffraction in elastic media under actions of concentrated and distributed sources of different form. Theoretical studies of such problems based on classical methods of mathematical physics have sufficiently extensive bibliography [1-3].

Real solid massif, besides elastic properties, possesses the whole spectrum of other properties, which have an essential effect on processes of seismic wave propagation and stress-strained state of media. In this connection the complication of mathematical model is absolutely necessary taking into account the more complete operative factors during the study of seismic processes. The one of these factors is the temperature of a massif, which substantially influences on its stress-strained state by static and dynamic effects. Investigation of dynamics of thermoelastic medium under the actions of non-stationary power and thermal sources relates to the poorly studied problems of Mechanics and Mathematical Physics. The research of Novacki [4], Kupradze et al., [5], Kech and Teodorescu [6] were coming to be regarded as a major contribution to the study of thermoelastic problems.

In connection with a complexity of constructing the solutions of the system of thermo-elastodynamics equations (its form relates to the systems of mixed hyperbolic-parabolic type), regularly these equations are simplified with disregarding the action of elastic deformations on the temperature field of medium. For this model, which are called uncoupled thermoelasticity, at first it is possible to define temperature field, solving the well studied heat conduction equation, then to define displacements and speeds of medium the classical equations of elastic body dynamics are used. The gradient of temperature field enters into as the mass force. But even for such mathematical model the class of solved problems is very limited.

In this article we consider the thermoelastic state of semi-plane with the cylindrical cavity under an action of non-stationary mass powers and thermal sources by using the model of

*ORCID: http://orcid.org/0000-0003-0915-2759 
coupled thermoelasticity. Thus we take into account the action of elastic deformation velocity on an temperature field. We develop boundary element method (BEM) for this problem based on generalized functions method (GFM) of construction of boundary value problem (BVP) solutions for differential equations.

\section{MOTION EQUATIONS OF THERMOELASTIC MEDIA}

The isotropic thermoelastic medium is characterized by the finite numbers of thermodynamic parameters: mass density $\rho$, elastic Lame constant $\lambda$ and $\mu$, thermoelastic and thermal constants $\gamma, \eta, \kappa$. All constants are positive.

Dynamics of thermoelastic medium in the Cartesian coordinates system is described by the system of differential equations of mixed hyperbolic-parabolic type [7]:

$$
\begin{aligned}
& \sigma_{i j, j}-\rho \ddot{u}_{i}+F_{i}=0, \\
& \theta_{, j j}-\kappa \dot{\theta}-\eta \dot{u}_{j, j}+Q=0, \quad j=1, \ldots, N
\end{aligned}
$$

where $u_{i}(x, t), \sigma_{i j}(x, t)$ are the components of the displacement vector and stress tensor, $u_{3}(x$, $t)=\theta(x, t)$ is temperature; $\mathrm{F}=\mathrm{F}_{i} e_{i}$ is the mass force; $Q$ is the power of thermal source, $\lambda, \mu$ are elastic Lame parameters; $\gamma \equiv(3 \lambda+2 \mu) \alpha_{t}, \alpha_{t}$ is coefficient of linear thermal expansion, $\kappa=\lambda_{0} / b_{\varepsilon}$ is coefficient of thermal diffusivity, $\lambda_{0}$ is the coefficient of thermal conductivity, $\eta \equiv \frac{\gamma T_{0}}{\lambda_{\mathrm{o}}}, T_{0}$ is the current absolute temperature of the medium in the initial state. The stress tensor $\sigma_{i j}$ is connected with displacements and temperature by the Duhamel-Neumann law:

$$
\sigma_{i j}=\lambda \delta_{i j} \delta_{k l} u_{k},{ }_{l}+\mu\left(u_{i},{ }_{j}+u_{j},_{i}\right)-\gamma \delta_{i j} \theta
$$

We will use its general form

$$
\begin{gathered}
\sigma_{i j}=C_{i j}^{k l} u_{k}{ }_{l}-\gamma \delta_{i j} \theta, \\
C_{i j}^{k l}=\lambda \delta_{i j} \delta_{k l}+\mu\left(\delta_{i k} \delta_{j l}+\delta_{i l} \delta_{j k}\right)
\end{gathered}
$$

Here $\delta_{i j}$ is Kronecker's symbol. The partial derivatives on the appropriate coordinates are designated by symbol after comma: $u_{i, j} \equiv \partial u_{i} \partial x_{j}$, differentiation with respect to time $t: \stackrel{\circ}{u}=\partial \mathrm{u} / \partial \mathrm{t}$.

Here we consider the case of plane deformation: $x=\left(x_{1}, x_{2}\right), i, j=1,2$. Everywhere the tensor convolutions are taken on the similar indices.

\section{THE STATEMENT OF THE BOUNDARY VALUE PROBLEM FOR A THERMOELASTIC SEMI-PLANE WITH CAVITY}

Let consider the thermoelastic semi-plane $\Pi^{+}=\left\{x_{1}>0,-\infty<x_{2}<\infty\right\}$ (the boundary $\Pi$ ) with cylindrical cavity $S^{+}, S$ is an boundary of $S^{+}, S^{-}$is region of elastic media without its boundary, $\Pi+S=B, n(x)$ is external normal to $B$. We suppose that acting forces $F(x, t)$ and thermal source $Q(x, t)$ are known and the initial state of medium is known also:

$$
u_{i}(x, 0)=0, \theta(x, 0)=\theta^{0}(x), x \in S^{-}+B ; \quad \dot{u}_{i}(x, 0)=0, x \in S^{-}
$$

On the boundary of cavity the stresses and heat flow are given:

$$
\sigma_{i j}(x, t) n_{j}(x)=q_{i}^{S}(x, t), \quad \frac{\partial \theta(x, t)}{\partial n}=\theta^{S}(x, t), \quad x \in S
$$


The boundary of semi-space is free from loads and heat fluxes:

$$
\sigma_{i j}(x, t)=0, \quad j=1,2, \frac{\partial \theta(x, t)}{\partial n}=0, \quad x_{1}=0
$$

The system (1) with use (2) can be written in the closed form

$$
\begin{gathered}
L_{i j}\left(\partial_{x}, \partial_{t}\right) u_{j}+F_{i}=0 \\
L_{i j}=(\lambda+\mu) \partial_{i} \partial_{j}+\left(\mu \Delta-\rho \partial_{t} \partial_{t}\right) \delta_{i j}-\gamma \delta_{j(N+1)} \partial_{i}, \quad i=1,2 \\
L_{3 j}=\left(\Delta-\kappa^{-1} \partial_{t}\right) \delta_{j(N+1)}-\eta\left(1-\delta_{j(N+1)}\right) \partial_{t} \partial_{j}, \quad 1,2,3
\end{gathered}
$$

It is the system of mixed hyperbolic-parabolic type. In this case there are thermoelastic shock waves with the jump of stresses, speeds and heat fluxes at their fronts appeared in the media $[8,9]$. The solutions satisfy to the next conditions on the jumps at the wave fronts $F_{t}$ :

$$
\begin{gathered}
{[u]_{F_{t}}=0, \quad\left[\sigma_{i j} n_{j}+\rho c_{k} \dot{u}_{i}\right]_{F_{t}}=0,} \\
{[\theta]_{F_{t}}=0, \quad[\dot{\theta}]_{F_{t}}=\frac{p \kappa n_{j}}{c_{k}}\left[\dot{u}_{j}\right]_{F_{t}} \quad k=1,2}
\end{gathered}
$$

Here $n=\left\{n_{1}, n_{2}\right\}$ is vector of unit normal to wave front $F_{t}$ which propagates in a thermoelastic medium with one of the following velocities of thermoelastic waves:

$$
c_{1}=\sqrt{\frac{\lambda+2 \mu}{\rho}}, \quad c_{2}=\sqrt{\frac{\mu}{\rho}},
$$

These velocities coincide with velocity of propagation of dilatation and shear waves in elastic media.

It is necessary to define the displacements, temperature and stresses of thermoelastic semiplane with cavity which must to satisfy the conditions of radiation on infinity.

\section{GENERALIZED FUNCTION METHOD. DYNAMIC ANALOGUE OF SOMIGLIANA FORMULAE IN DISTRIBUTION SPACE}

We'll consider BVP in the space of Laplace transformation of displacements in time t:

$$
u(x, p)=\int_{0}^{\infty} u(x, t) e^{-p t} d t
$$

Here we use the Generalized Function Method (GFM) to construct the generalized solution of this problem [10].

At first we introduce the general regular vector-functions:

$$
\hat{u}(x, p)=u(x, p) H_{B}^{-}(x), \hat{F}=F(x, p) H_{B}^{-}(x), \hat{Q}=Q(x, p) H_{B}^{-}(x),
$$

where $H_{B}^{-}(x)$ is characteristic function of the region $S^{-}$:

$$
H_{B}^{-}(x)= \begin{cases}1, & x \in S^{-} \\ 0,5, & x \in B \\ 0, & x \notin S^{-}+B\end{cases}
$$


Note, that generalized derivative of such functions is

$$
\partial_{j} \hat{f}=H_{B}^{-}(x) \partial_{j} f-n_{j} f \delta_{B}(x),
$$

where $n_{j} f \delta_{S}(x)$ is a singular function - simple layer on surface $B$.

By using generalized differentiation, we obtain singular distributions and their derivatives as simple layers in right part:

$$
L_{i j}\left(\partial_{x}, p\right) \hat{u}_{j}(x, p)=-\bar{F}_{i}(x, p) H_{B}^{-}(x)-\hat{\mathrm{P}}_{i}^{B}(x, p)
$$

We obtain the densities of (8) if these simple layers depend on boundary displacements, temperatures, stresses and heat flows [11]. The shock waves are the generalized solutions of equations. The conditions on the wave front (7) give zero densities on the simple and double layers, which appear by generalized differentiation of shock thermoelastic waves. Therefore they disappeared in the eqn (8).

By using of the Laplace transformation of Green's tensor for the free thermoelastic semiplane $\vec{V}_{m}^{i}(x, p)$, the convolution of right part (8) gives the expression of generalized solution of this problems:

Theorem 1. If $u(x, p)$ is classic solutions of $B V P$, then generalized solution of $B V P$ has the form of following convolution

$$
\begin{gathered}
\hat{u}_{m}(x, p)=\vec{V}_{m}^{i} *\left(\rho \hat{F}_{i}+\hat{G}_{i}\right)+\vec{V}_{m}^{i} * \bar{q}_{i}^{s} \delta_{S}(x)+C_{i j}^{k l} \vec{V}_{m}^{i},{ }_{j} *\left(\bar{u}_{k}^{B} n_{l} \delta_{S}(x)\right)+ \\
+\vec{V}_{m}^{N+1} *\left(\kappa^{-1} \hat{F}_{N+1}+\hat{G}_{N+1}\right)+\vec{V}_{m}^{N+1} *\left(\bar{q}^{S}-\eta p \bar{u}_{j}^{B} n_{j}\right) \delta_{s}(x)+\vec{V}_{m}^{N+1},_{j} *\left(\bar{\theta}^{B} n_{j} \delta_{S}\right) \\
i, j, k, l=1,2, m=1, \ldots, 3
\end{gathered}
$$

Here we use the boundary conditions (5) on the boundary of semi-plane.

\section{GREEN'S TENSOR FOR THE FREE THERMOELASTIC SEMI-PLANE AND FUNDAMENTAL TENSOR OF THERMO-STRESSES}

The fundamental solutions $V_{i}^{k}(x, t)$ of thermoelasticity (1) for the free semi-space was constructed in the paper [11]. This matrix of solutions satisfies equations under the action of the impulse concentrated mass forces and thermal sources, described by the singular $\delta$-functions:

$$
\begin{gathered}
L_{i j}\left(\partial_{1}, \partial_{2}, \partial_{t}\right) V_{j}^{k}(x, t)=\delta_{i}^{k} \delta(x) \delta(t) \\
L_{3 j}\left(\partial_{1}, \partial_{2}, \partial_{t}\right) V_{j}^{k}(x, t)=\delta_{i}^{3} \delta(x) \delta(t), \quad i=1,2, j . k=1,2,3
\end{gathered}
$$

There are the boundary conditions on the free surface:

$$
\begin{gathered}
\sum_{i 1}^{m}=\left(\lambda V_{k, k}^{m}-\gamma V_{3}^{m}\right) \delta_{i 1}+\mu\left(V_{i, 1}^{m}+V_{i, 1}^{m}\right)=0, \quad x_{1}=h \\
\frac{\partial V_{3}^{m}}{\partial x_{1}}=0
\end{gathered}
$$

here $\Sigma_{i j}^{m}$ is the sum of the stresses, generated by the fundamental solutions of the $\mathrm{V}$ by fixed $m$ :

$$
\sum_{i j}^{m}=\lambda V_{k, k}^{m} \delta_{i j}-\gamma V_{3}^{m} \delta_{i j}+\mu\left(V_{i, j}^{m}+V_{j, i}^{m}\right)
$$


The second condition (7) indicates the absence of heat flux on the border. It satisfies to the following conditions for radiation and damping at infinity also:

$$
\begin{gathered}
V_{i}^{k}(x, t)=0 \text { for } \forall t<0 \\
V_{i}^{k}(x, t) \rightarrow 0 \text { for } \forall t>0, x \rightarrow \infty
\end{gathered}
$$

Thus we specified some conditions for radiation, which describe reflected wave from the boundary of semi-plane. The analytic formulas for calculation have complex form [12, 13].

To express the formulae of Theorem 1 in integral form, we input the fundamental tensor of thermo-stresses $\left.T_{i}^{j}(x, n, t): i, j, k, l=\overline{1, N}, \quad m=1, N+1\right)$

$$
\bar{T}_{m}^{k}(x, n, p)=C_{i j}^{k l} \bar{V}_{m, j}^{i} n_{l}-\eta p \bar{V}_{m}^{3} n_{k}, \quad \bar{T}_{m}^{3}=\bar{V}_{m, j}^{3} n_{j}
$$

The following Theorems for this tensor are valid.

Theorem 2. The tensor $\bar{T}_{m}^{k}$ for fixed $k$ is fundamental solution of equations of thermoelasticity (6) by corresponding mass power source and thermal source of multipole type:

for $k=\overline{1, N}$

$$
\begin{gathered}
\bar{F}_{s}=\left(C_{s j}^{[k] l} n_{l} \partial_{j}-\eta p n_{k} \delta_{s}^{N+1}\right) \delta(x), \\
\bar{F}_{s}=\delta_{s}^{[N+1]} n_{j} \delta,_{j} \quad(s=\overline{1, N+1}, \quad j, l=\overline{1, N}) .
\end{gathered}
$$$$
\text { for } k=N+1
$$

Theorem 3. (Dynamical analogue of Gauss Formulae). If $D^{-} \in S^{-}+B$, and boundary $D$ is Lyapunov's surface [8], then

$$
\begin{aligned}
& V . P . \int_{D} \bar{T}_{i}^{m}(x-y, n(y), p) d S(y)+\rho p^{2} \int_{D^{-}} \bar{V}_{i}^{m}(x-y, p) d V(y)=\delta_{i}^{m} H_{D}^{-}(x) \\
& V . P . \int_{D}^{T_{N+1}^{m}}(x-y, n(y), p) d S(y)+p \kappa^{-1} \int_{D^{-}} \bar{V}_{N+1}^{m}(x-y, p) d V(y)=\delta_{N+1}^{m} H_{D}^{-}(x)
\end{aligned}
$$

where in all integrals are regular for inner points of $\mathrm{D}$, and are taken as Value Principal on boundary.

\section{THE SOLUTION OF BVP. SINGULAR BOUNDARY INTEGRAL EQUATIONS}

We can write the formula (9) in integral form using the fundamental tensors.

Theorem 4. If classic solution $\vec{u}(x, p)$ of BVP exists then it could be expressed in the following regular integral form:

$$
\begin{gathered}
\bar{u}_{m} H_{B}^{-}(x)=\bar{V}_{m}^{j} *\left(\bar{F}_{j}+\bar{G}_{j}\right)+ \\
+\int_{s}\left(\bar{V}_{m}^{k}(x-y, p) \bar{q}_{k}^{S}(y, p)+\bar{T}_{m}^{k}(x-y, p, n(y)) \bar{u}_{k}^{S}(y, p)\right) d S(y)
\end{gathered}
$$

for $x \in S^{-}+\Pi$. Here $\bar{q}_{3}^{S}=\bar{q}^{S}(x, p), \quad u_{3}^{S}=\bar{\theta}^{S}(x, p)$.

We note that the equation's terms with displacements and temperature in formula (9) disappear on the boundary of the semi-plane, since the corresponding integral kernels are equal zero. Only the integrals on the cavity boundary remain.

The formula of the Theorem 4 expresses the displacements and the temperature inside the area through their boundary values, as well as the values of the load and the heat flux at the boundary. It is an analogue of the Somigliana Formulae of the static theory of elasticity, which expresses the displacement of an elastic medium through the boundary values of load and displacement [14]. Therefore, this formula can be called the thermoelastic Analogue of the Formulae of Somigliana $[15,16]$. 
Since only a part of the boundary functions is known, the Formulae do not give a solution of the BVP. However, it is also valid for $x \in S$ in the sense of definition of $H_{S}^{-}(x)$ and gives the boundary integral equations for solving the BVPs. So, using Theorem 4 , we can prove the following Theorem 5 .

Theorem 5. If classic solution of $B V P \vec{u}(x, p)$ satisfies Helder's condition on the boundary for any $p(\operatorname{Re} p>0)$, then

$$
\begin{aligned}
& 0,5 \bar{u}_{m}=\bar{V}_{m}^{j} *\left(\bar{F}_{j}+\bar{G}_{j}\right)+\int_{S}\left(\bar{V}_{m}^{k}(x-y, p) \bar{q}_{k}^{S}(y, p)\right) d S(y)+ \\
& +V . P . \int_{S}\left(\bar{T}_{m}^{k}(x-y, p, n(y)) \bar{u}_{k}^{S}(y, p)\right) d S(y), \quad m, k=1,2,3
\end{aligned}
$$

Here $\bar{q}_{3}^{S}=\bar{q}^{S}(x, p), u_{3}^{S}=\bar{\theta}^{S}(x, p)$. For $x \in S$ second integral is singular, it exists in Value Principle sense.

\section{CONCLUSION}

The boundary integral equations (BIE) allow finding unknown boundary functions, and obtained formulas of Theorem 5 are valid to find the needed solution. All received equations are amenable to a numerical solution based on the Boundary Element Method (BEM).

The algorithm of numerical realization of the Method of Boundary Integral Equations (BIEM) for the solution of plane BVPs $\left(R^{2} \times t\right)$ of coupled thermoelastodynamics is being developed. A numerical program for calculating the integral kernels of a system of BIEs in the space of Laplace transformation in time and a numerical program for calculating the first approximation of a system of singular BIEs for determining boundary displacements and temperature have been developed.

\section{REFERENCES}

[1] Aitaliev, Sh.M., Alexeyeva, L.A, Dildabaev, Sh.M. \& Zhanbyrbaev, N.B., Method of boundary integral equations in the problems of dynamics of elastic multicoupled solids - Almaty: "Nauka"-1992.

[2] Guz, A.N., Kubenko, V.D. \& Cherevko, M.A., Diffraction of elastic waves, Kiev: "Naukova Dumka", 1978.

[3] Erzhanov, Zh.S., Aitaliev, Sh.M. \& Alexeyeva, L.A., Dynamics of tonnels and underground pipelines.- Almaty: "Nauka", 1989.

[4] Novacki, V., Theory of elasticity. Moscow: "Mir", 1975.

[5] Kupradze, V.D., Gegelia, T.G., Basheleishvili, E.O. \& Burchuladze, T.V., 3D problems of mathematical theory of elasticity and thermoelasticity. Moscow: "Nauka", 1976.

[6] Kech, V. \& Teodorescu, P., Introduction into theory of generalized functions with applications in technics- Moscow: "Mir", 1978.

[7] Novacki, V., Dynamical problems of thermoelasticity. Moscow: "Mir", 1970.

[8] Alexeyeva, L.A. \& Dadaeva, A.N., About uniqueness of solutions of boundary-valued problems of thermoelasticity with considering of thermal shoch waves. Proceedings of KazNTU, Series of Mathematics, Mechanics and Informatics, 28, pp. 11-18, 2013. 
[9] Alipova, B.N., Alexeyeva, L.A. \& Dadayeva, A.N., Shock waves as generalized solutions of thermoelastodynamics equations. On the uniqueness of boundary value problems solutions. AIP Conference Proceedings, ICNPAA 2016 World Congress 11th International Conference on Mathematical Problems in Engineering, Aerospace and Sciences, July 4-8, 2016, La Rochelle, France, American Institute of Physics, 2017. https://doi.org/10.1063/1.4765466

[10] Vladimirov, V.S., Generalized functions in mathematical physics. Moscow: "Nauka", 1978.

[11] Alexeyeva, L.A. \& Kupesova (Alipova), B.N., Method of generalized functions for boundary problems of coupled thermoelastodynamics. Journal of Applied Mathematics and Mechanics (of Russian Academy of Sciences), 65(2), pp. 334-345, 2001. https://doi.org/10.1016/s0021-8928(01)00037-5

[12] Alexeyeva, L.A. \& Alipova B.N., Tensor of Green for thermoelastic semi-plane with free boundary. Mathematical Journal of RK, 17(1), pp. 32-42, 2017.

[13] Alipova, B.N., Method of Boundary Integral Equations (BIEM) and generalized solutions of transient problems of thermoelastodynamics. ICNPAA 2012, 9-15 July, AIP Conference Proceedings, American Institute of Physics, Vien, Austria, 1493, 39, pp. 39-46, 2012.

https://doi.org/10.1063/1.4765466

[14] Alipova, B., Dynamical analogue of formulae of Gauss of transient problems of thermoelastodynamics. AIP Conf. Proc. 1637, 26 (2014); 1 10th International Conference On Mathematical Problems In Engineering, Aerospace And Sciences: ICNPAA 2014, Narvik, Norway, 15-18 July 2014. https://doi.org/10.1063/1.4904561

[15] Alipova, B., Fundamental tensor of tension of coupled thermoelastodynamics in 2-D and 3-D cases. Journal |MESA, [S.1.], 7(3), pp. 513-524, 2016. Available at: http:// nonlinearstudies.com/index.php/mesa/article/view/1179

[16] Alipova, B., Certificate of Copyright of intellectual property No 001494 dd 10/23/2013 "Dynamical analogue of Somigliana's Formulae of coupled thermoelastodynamics". Available at: http://www.avtor.uz/index.php?option-com_desposition\&task=display_ desp_det\&id=1600\&itemid=88\&lang=ru 